\title{
Flexibility-induced effects in the Brownian motion of colloidal trimers
}

\author{
Ruben W. Verweij $\odot,{ }^{1, *}$ Pepijn G. Moerman $\odot,{ }^{2,3,}{ }^{*}$ Nathalie E. G. Ligthart, ${ }^{2}$ Loes P. P. Huijnen, ${ }^{1}$ Jan Groenewold $\odot,{ }^{2,4}$ \\ Willem K. Kegel $\odot,{ }^{2, \dagger}$ Alfons van Blaaderen, ${ }^{3, *}$ and Daniela J. Kraft ${ }^{1, \S}$ \\ ${ }^{1}$ Huygens-Kamerlingh Onnes Lab, Universiteit Leiden, 2333 CA Leiden, The Netherlands \\ ${ }^{2}$ Debye Institute for Nanomaterials Research, Department of Chemistry, Utrecht University, 3584 CC Utrecht, The Netherlands \\ ${ }^{3}$ Debye Institute for Nanomaterials Research, Department of Physics, Utrecht University, 3584 CC Utrecht, The Netherlands \\ ${ }^{4}$ Academy of Advanced Optoelectronics, South China Normal University, Guangzhou 510006, China
}

(Received 8 March 2020; revised 6 April 2020; accepted 12 June 2020; published 24 July 2020)

\begin{abstract}
Shape changes resulting from segmental flexibility are ubiquitous in molecular and biological systems, and are expected to affect both the diffusive motion and (biological) function of dispersed objects. The recent development of colloidal structures with freely jointed bonds have now made a direct experimental investigation of diffusive shape-changing objects possible. Here, we show the effect of segmental flexibility on the simplest possible model system, a freely jointed cluster of three spherical particles, and validate long-standing theoretical predictions. We find that, in addition to the rotational diffusion time, an analogous conformational diffusion time governs the relaxation of the diffusive motion, unique to flexible assemblies, and that their translational diffusivity differs by a small but measurable amount. We also uncovered a Brownian quasiscallop mode, where diffusive motion is coupled to Brownian shape changes. Our findings could have implications for molecular and biological systems where diffusion plays an important role, such as functional site availability in lock-and-key protein interactions.
\end{abstract}

DOI: 10.1103/PhysRevResearch.2.033136

\section{INTRODUCTION}

Many (macro)molecular systems display segmental flexibility, e.g., biopolymers such as transfer RNA [1], intrinsically disordered proteins [2], myosin [1], immunoglobulins [1], and other antibodies [3-6]. For most of these systems, the flexibility not only affects the motion of the complex but also its (biological) function [3,4,7-9]. For example, proteins often function through shape-dependent lock-and-key interactions where active sites of enzymes are reshaped during the interaction, leading to an induced fit [10]. Additionally, enzymes like adenylate kinase can accelerate biochemical reactions with remarkable specificity and efficacy thanks to a flexible "lid" that opens and closes at each reaction cycle. Because shape has a large effect on the diffusive motion of structures at the short timescales relevant to these reactions, it is expected that the diffusion of reconfigurable objects is different from rigid ones [1,11-13]. Moreover, Adeleke-Larodo et al. recently proposed [14] that changes in an enzymes flexibility upon substrate binding could be responsible for the observed enhanced diffusion of active enzymes [15,16]. Therefore, a rigorous understanding of enzyme function and diffusion requires quantitative knowledge of protein flexibility [17].

\footnotetext{
*These authors contributed equally to this work.

†W.K.Kegel@uu.nl

¥A.vanBlaaderen@uu.nl

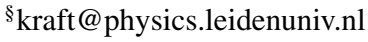

Published by the American Physical Society under the terms of the Creative Commons Attribution 4.0 International license. Further distribution of this work must maintain attribution to the author(s) and the published article's title, journal citation, and DOI.
However, direct experimental measurements of flexibility in molecular systems are challenging because they require single-molecule measurement techniques with high spatial and temporal resolution. One way to circumvent this problem is to employ colloidal particles, which have been used as model systems for (macro)molecular structures [18-20], because of their unique combination of microscopic size and sensitivity to thermal fluctuations. Studies on the Brownian motion of rigid colloids of various shapes such as ellipsoids [21-23], boomerangs [24-26], and clusters $[27,28]$ revealed that shape affects the diffusive motion at short timescales. Additionally, displacements are larger in directions that correspond to smaller hydrodynamic drag [21,24,25,27-29] and different diffusive modes can be coupled, e.g., helical particles rotate as they translate and vice versa [30]. At longer timescales, the influence of particle shape decreases because of rotational diffusion [21].

While rigid assemblies have been extensively studied, little is known about the effect of flexibility. In this work, we numerically and experimentally investigate the effect of segmental flexibility on the simplest possible model system: a freely jointed chain of three spherical colloidal particles, called flexible trimers or "trumbbells" [31,32]. To describe the diffusion of such segmentally flexible objects numerical models were proposed in the 1980s $[31,33,34]$ and the long time diffusive motion was predicted to be determined by the shape average of the instantaneous diffusivities (so-called rigid-body approximation) $[4,35,36]$. Here, we test these models for the first time using direct experimental measurements of the diffusion of colloidal particles. These measurements were made possible by the recent development of colloidal structures with freely jointed bonds [37-43], and flexible chains [44]. We extract the short-time diffusion tensor of flexible trimer chains 
from their motion and find good agreement with numerical calculations. We furthermore uncovered a Brownian quasiscallop mode, where diffusive motion is coupled to Brownian shape changes. We compare our results to rigid trimers frozen in a particular shape and find that their translational diffusivity differs by a small, but measurable amount. Finally, we consider the diffusive behavior at longer timescales. We find that, in addition to the rotational diffusion time, an analogous conformational diffusion time governs the relaxation of the diffusive motion, which is unique to flexible assemblies.

\section{METHODS}

\section{A. Experiment}

Flexible clusters of three colloidal supported lipid bilayers (CSLBs) were prepared as described in previous work $[37-39,43]$. To test the generality of the results presented here, we used two particle sizes, namely $1.93 \mu \mathrm{m}$ and $2.12 \mu \mathrm{m}$ silica particles, with different methods of functionalization.

The CSLBs consisting of $2.12 \mu \mathrm{m}$ silica particles were prepared as described in our recent work [43]. Briefly, the particles were coated with a fluid lipid bilayer by deposition of small unilamellar vesicles consisting of $98.8 \mathrm{~mol} \%$ DOPC ( $(\Delta 9$-Cis $) \quad$ 1,2-dioleoyl-sn-glycero-3-phosphocholine), $1 \mathrm{~mol} \%$ DOPE-PEG(2000) (1,2-dioleoyl-sn-glycero-3phosphoethanolamine- $\mathrm{N}-[$ methoxy(polyethylene glycol)2000]) and $0.2 \mathrm{~mol} \% \quad$ TopFluor-Cholesterol (3(dipyrrometheneboron difluoride)-24-norcholesterol) or DOPE-Rhodamine (1,2-dioleoyl-sn-glycero-3phosphoethanolamine-N-(lissaminerhodamine B sulfonyl)). The bilayer coating was performed in a buffer at $\mathrm{pH}$ 7.4 containing $50 \mathrm{mM}$ sodium chloride $(\mathrm{NaCl})$ and $10 \mathrm{mM}$ 4-(2-Hydroxyethyl)-1-piperazineethanesulfonic acid (HEPES). We added double-stranded DNA (of, respectively, strands DS-H-A and DS-H-B, see Supplemental Material, Table S1, Ref. [45]) with an 11 base pair long sticky end and a double stearyl anchor, which inserts itself into the bilayer via hydrophobic interactions [see Fig. 1(b)]. When two particles with complementary DNA linkers come into contact, the sticky ends hybridize and a bond is formed. Self-assembly experiments were performed in a different buffer of $\mathrm{pH} 7.4$, containing $200 \mathrm{mM} \mathrm{NaCl}$ and $10 \mathrm{~mm}$ HEPES. We imaged 21 trimers of $2.12 \mu \mathrm{m}$ CSLBs, that were formed by self-assembly in a sample holder made of polyacrylamide (PAA) coated cover glass. The PAA functionalization was carried out using a protocol [46] which we modified by adding $0.008 \mathrm{~mol} \%$ bisacrylamide and performing the coating under a nitrogen atmosphere, both of which resulted in a more stable coating. Using an optical microscope, we imaged the clusters for $5 \mathrm{~min}$ at frame rates between 5 to $10 \mathrm{fps}$. Particle positions were tracked using a custom algorithm [43] available in TRACKPY by using the locate_brightfield_ring function [47].

Additionally, we analyzed nine trimers of $1.93 \mu \mathrm{m}$ CSLBs, with silica particles purchased from Microparticles $\mathrm{GmbH}$ (product code $\mathrm{SiO}_{2}-\mathrm{R}-\mathrm{B} 1072$ ). For these particles, we used a similar protocol to form supported lipid bilayers with only two minor modifications: first, the lipid composition we used was $98.9 \mathrm{~mol} \%$ DOPC, $1 \mathrm{~mol} \%$ DOPE-PEG(2000) and $0.1 \mathrm{~mol} \%$ DOPE-Rhodamine. Second, we added Cy3-labeled DNA with a self-complementary 12 base pair sticky end and a cholesterol
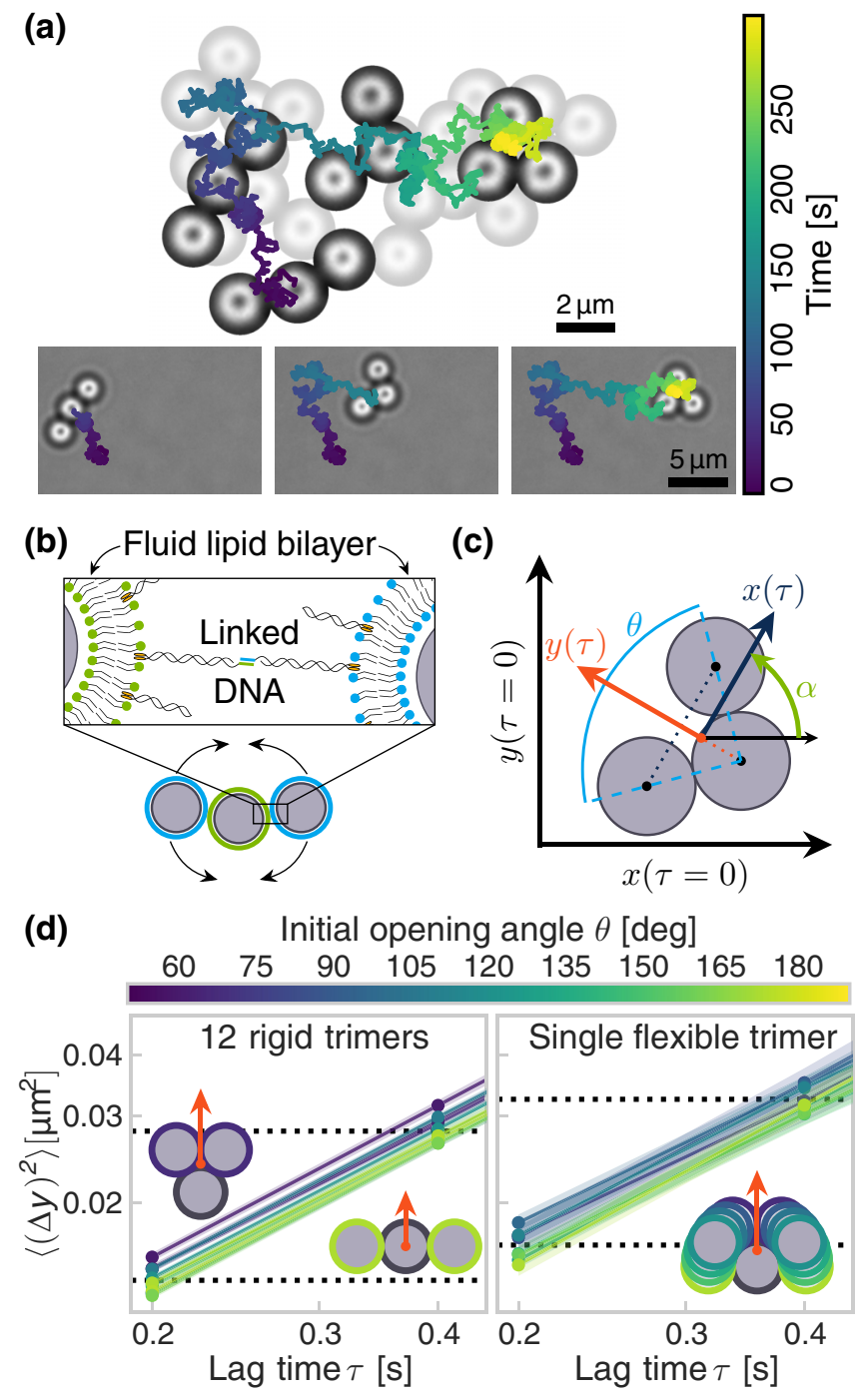

FIG. 1. Diffusion of flexible trimers. (a) Overlay of bright-field microscopy images of a flexible trimer with the position of its center of mass as function of time. (b) Schematic (not to scale) of flexible trimers that are self-assembled from colloid supported lipid bilayers. We inserted DNA linkers into the fluid lipid bilayer surrounding the particle, resulting in bonded particles that can rearrange with respect to each other. (c) Illustration of the body-centered coordinate system. (d) The mean squared displacement of rigid and flexible trimers. The translational mean-squared displacement of flexible trimers in the $y$-direction is angle dependent for short lag times, at longer lag times this angle dependence is no longer present due to rotational and conformational relaxation, which happens on a shorter timescale than for rigid trimers (raw data, not scaled with friction coefficients).

anchor that inserts itself into the lipid bilayer due to hydrophobic interactions. We used the DNA sequence from Leunissen et al. [48] (see Supplemental Material, Table S1, Ref. [45], strands PA-A and PA-B).

To image the $1.93 \mu \mathrm{m}$ CSLBs we used a flow cell produced as detailed in the Supplementary Material of Montanarella et al. [49] As the base of our flow cell we used a single capillary with dimensions $3 \mathrm{~cm} \times 2 \mathrm{~mm} \times 200 \mu \mathrm{m}$. To prevent the lipid coated clusters from sticking to the class capillary, we coated the inside of the capillary with 
poly(2-hydroxyethyl acrylate) (pHEA) polymers. To this end, we first flushed the cell with consecutively $2 \mathrm{~mL} 2 \mathrm{mM}$ $\mathrm{NaOH}$ solution, $2 \mathrm{~mL}$ water, and $2 \mathrm{~mL}$ EtOH. We then functionalized the glass surface with the silane coupling agent 3-(methoxysilyl)propyl methacrylate (TPM) by filling the flow cell with a mixture of $1 \mathrm{~mL} \mathrm{EtOH}, 25 \mu \mathrm{L}$ TPM, and $5 \mu \mathrm{L} 25 \mathrm{vol} \% \mathrm{NH}_{3}$ in water and leaving it for $1 \mathrm{~h}$. We then washed and dried the flow cell by flushing with $2 \mathrm{~mL}$ ethanol and subsequently with nitrogen. We grew pHEA brushes from the surface through a radical polymerization by filling the cell with a mixture of $2.5 \mathrm{~mL}$ EtOH, $500 \mu \mathrm{L}$ HEA, and $20 \mu \mathrm{L}$ Darocur 1173 photoinitiator. We initiated the reaction by placing the cell under a UV lamp $\lambda=360 \mathrm{~nm}$ for $10 \mathrm{~min}$. Finally, we flushed the cells with $10 \mathrm{~mL} \mathrm{EtOH}$ or Millipore filtered water. We stored the coated cells filled with $\mathrm{EtOH}$ or Millipore filtered water and for no more than 1 day. Self-assembly experiments were performed in a buffer of $\mathrm{pH}$ 7.4 , containing $50 \mathrm{~mm} \mathrm{NaCl}$ and $10 \mathrm{~mm}$ HEPES. We imaged nine freely jointed trimers and 13 rigid trimers stuck in various opening angles shown in Fig. S6 of the Supplemental Material, Ref. [45] for $30 \mathrm{~min}$ with a frame rate of five frames per second. Particle positions were tracked using the 2007 MATLAB implementation by Blair and Dufresne of the Crocker and Grier tracking code [50].

\section{B. Diffusion analysis}

For all analyses, we only selected trimers that showed all bond angles during the measurement time, experienced no drift, and were not stuck to the substrate. After the particle positions were tracked, we determined the short-time diffusivity of the trimers as described by Eq. (3).

The three friction correction factors that account for substrate friction were determined in the following way:

$$
\begin{aligned}
\phi_{t t} & =\left\langle\boldsymbol{D}[t t]_{t} /\left(\sigma_{e} \boldsymbol{D}[t t]_{e, 0}\right)\right\rangle, \\
\phi_{(\alpha \alpha, \theta \theta)} & =\left\langle\boldsymbol{D}[(\alpha \alpha, \theta \theta)]_{t} /\left(\sigma_{e}^{3} \boldsymbol{D}[(\alpha \alpha, \theta \theta)]_{e, 0}\right)\right\rangle, \\
\phi_{i j} & =\sqrt{\phi_{i i} \phi_{j j}} \quad \text { for } \quad i \neq j,
\end{aligned}
$$

where $\boldsymbol{D}[i j]_{k}$ denotes the theoretical $(k=t)$ or experimental $(k=e)$ diffusion tensor element and $\sigma_{e}$ the experimental particle radius. The subscript $t t$ denotes the translational component of the diffusivity. These factors were determined separately for each experiment because differences in surface and particle functionalizations resulted in differences in substrate-particle and particle-particle friction, that in turn affected the diffusivity of the cluster. We separated the correction factors into these three factors because different modes of diffusion were expected to lead to different amounts of friction with the substrate [51].

We calculated the elements of the diffusion tensor given in Eq. (3) separately for all trimers. For each pair of frames, we determined the initial average opening angle $\bar{\theta}$ of the trimer between $t$ and $t+\tau_{\text {short }}$, with $\tau_{\text {short }}=0.25 \mathrm{~s}$. Then, we stored the diffusion tensor elements separately for each initial opening angle. For short times up to $\tau_{\text {short }}=0.25 \mathrm{~s}$, we used a bin size of $15^{\circ}$ while for longer times, we used two bins of $60^{\circ}$ covering the range of $\left[60^{\circ}, 120^{\circ}\right)$ and $\left[120^{\circ}, 180^{\circ}\right]$.
We scaled each element with the friction factors we obtained for that measurement, based on the diffusion coefficient for lag times up to $\tau_{\text {short. }}$. The average diffusion tensor elements were then obtained by fitting the overall slope of the mean (squared) displacements of all the individual diffusion tensor elements as a function of lag time [see Figs. 3(a), 3(c) 3(e) and Figs. 4(a) and 4(c)]. We used a linear function (with zero intercept) divided into ten segments with slopes $2 D_{i}$ (spaced evenly on a log scale), which correspond to the $i$ th diffusion coefficient for those lag times. This resulted in the average diffusion tensor for all binned average opening angles $\bar{\theta}$ as a function of the lag time $\tau$. For fitting, we used a standard leastsquares method and we estimated the error using a Bayesian method to find an estimate of the posterior probability distribution, by using a Markov chain Monte Carlo (MCMC) approach as implemented in the PYTHON packages LMFIT [52] and ECMEE [53]. We estimated the autocorrelation time $\tau_{\text {acor }}$ of the chain using the built-in methods and ran the analysis for at least $100 \tau_{\text {acor }}$ steps, where we discarded the first $2 \tau_{\text {acor }}$ steps (corresponding to a burn-in phase) and subsequently used every other $\tau_{\text {acor }} / 2$ steps (known as thinning). The reported values correspond to the maximum likelihood estimate of the resulting MCMC chain, the reported uncertainties correspond to the minimum and maximum of the obtained posterior probability distribution.

\section{Hydrodynamic modeling}

The diffusion of segmentally flexible objects can be described using hydrodynamic modeling [31,54]. To compare our experimental results to these predictions, we followed the procedure described by Harvey and coworkers. Of the seven degrees of freedom in three dimensions (three translational, three rotational, one internal degree of freedom) [31], we considered only the four degrees of freedom of interest for our quasi-two-dimensional system of sedimented clusters. Briefly, following the method outlined by Harvey and coworkers [31], we determined the hydrodynamic resistance (or friction) tensor $\boldsymbol{R}_{0}$ with respect to the central particle. Using this resistance tensor, we calculated the diffusion tensor $\boldsymbol{D}_{0}=k T \boldsymbol{R}_{0}^{-1}$, to which we applied the appropriate coordinate transformation to obtain the $7 \times 7$ diffusion tensor $\boldsymbol{D}_{\text {com }}$ relative to the center of mass of the cluster. We chose the center of mass as reference point because this is the best approximation of the center of diffusion of a flexible particle: in fact, it was found to be a better choice than either the center of diffusion or resistance of a rigid cluster of the same shape [31]. We also calculated the diffusion tensor with respect to the central particle and these results are shown in Sec. A of the Supplemental Material Ref. [45].

The diffusivity of flexible colloidal clusters can be modelled using bead or bead-shell models [55] and we employed both methods. For the bead model, we modelled the trimer using three beads (diameter of $2 \mu \mathrm{m}$ ) and for the bead-shell model, we modelled the trimer using approximately 2500 to 9500 smaller beads with bead radii from 54 to $31 \mathrm{~nm}$, respectively, where the beads where placed to form three $2 \mu \mathrm{m}$ shells. We followed existing methods [56,57] for constructing the bead-shell model: to summarize, the positions of the small beads were calculated by placing them on concentric circles, 
starting at the equator of an individual $2 \mu \mathrm{m}$ sphere and continuing the process towards the poles of the sphere using circles of decreasing radius and finally putting one sphere at each of the poles. Three spherical bead-shell models were then put together to form a trimer and we removed overlapping beads at the contact points between the particles. Examples of the model are shown in Fig. S1 of the Supplemental Material Ref. [45].

Because drag forces act on the surface of the particles, the bead-shell model is more accurate in describing the diffusive properties of the clusters [35,56,57]. The accurate consideration of hydrodynamic effects was found to be important for the segmentally flexible system we study: hydrodynamic interactions lead to a slower decay of the autocorrelation of the particle shape [58] and lead to an increase in the translational diffusivity $[1,4]$. We compared our experimental data to such a bead-shell model because it described our experimental data more accurately than the simple bead model, which is discussed in Sec. A of the Supplemental Material Ref. [45].

To calculate the diffusion tensor elements, we used the Rotne-Prager-Yamakawa (RPY) [59,60] interaction tensor $\boldsymbol{T}_{i j}$ to model hydrodynamic interactions between particles $i$ and $j$ :

$$
\boldsymbol{T}_{i j}=\frac{1}{8 \pi \eta_{0} R_{i j}}\left[\boldsymbol{I}+\frac{\boldsymbol{R}_{i j} \boldsymbol{R}_{i j}}{R_{i j}^{2}}+\frac{2 \sigma^{2}}{R_{i j}^{2}}\left(\frac{\boldsymbol{I}}{3}-\frac{\boldsymbol{R}_{i j} \boldsymbol{R}_{i j}}{R_{i j}^{2}}\right)\right],
$$

where $\sigma$ is the particle radius, $\boldsymbol{R}_{i j}$ is the vector between particles $i$ and $j, \boldsymbol{I}$ is the $3 \times 3$ identity matrix, and $\eta_{0}$ is the viscosity of the medium. Using the RPY tensor prevents singularities that may lead to the large, nonphysical numerical fluctuations [61] found when using lower-order terms (Oseen tensor), higher-order terms, or multibody effects [62].

We used the RPY tensor to model the hydrodynamic interactions between the beads and followed the procedure outlined by Harvey and coworkers [31] to obtain the diffusion tensor, as explained in the main text. This was done for all small bead radii and we used a linear extrapolation to zero bead size to obtain the final diffusion tensor elements [56,57]. Additionally, we used HYDROSUB [35] to model the diffusivity of rigid trimers of the same opening angles.

\section{RESULTS AND DISCUSSION}

To experimentally validate the theoretical predictions for the diffusion of segmentally flexible objects, we prepare flexibly linked colloidal trimers by self-assembly of colloid supported lipid bilayers [37-39,43]. Briefly, spherical colloidal silica particles are coated with a fluid lipid bilayer. DNA linkers with complementary sticky ends are inserted into the bilayer using a hydrophobic anchor. The particles are self-assembled by hybridization of the DNA sticky ends, which provide strong and specific interactions. The trimers are freely jointed because the DNA linkers can diffuse on the fluid lipid bilayer that surrounds the particles [see Fig. 1(b)]. The clusters undergo translational and rotational diffusion while they are also free to change their shape [see Fig. 1(a) and Supplementary Movie 1, Ref. [45]]. For simplicity, we used dense silica particles such that their mobility is confined to the bottom of the container by gravity, which leads to twodimensional Brownian motion.
For rigid objects in two dimensions, the diffusive motion can be described by a $3 \times 3$ diffusion tensor calculated from the linear increase of the mean squared displacements of the particle as function of lag time [63]. For flexible objects, this diffusion tensor has to be extended with an additional degree of freedom [31] for each internal deformation mode (here: one), and we therefore consider the $4 \times 4$ diffusion tensor $\boldsymbol{D}[i j]$. Here, $i, j \in[x, y, \alpha, \theta]$ are elements of a body-centered coordinate system [see Fig. 1(c)] at the center of mass. We chose the center of mass as reference point because, for flexible objects, it is more appropriate than either the center of diffusion or resistance of a rigid cluster of the same shape [31]. In this coordinate system the $y$-axis is perpendicular to the end-to-end vector and points away from the central particle, and the direction of the $x$-axis is chosen to form a right-handed coordinate system. We label the opening angle of the trimer $\theta$ and the (anticlockwise) rotation angle of the $x$-axis with respect to the laboratory frame $\alpha$. We align the laboratory frame such that it coincides with the body-centered coordinate system at $\tau=0$.

Shape determines the diffusion tensor for rigid objects and therefore we expect it to be important for flexible objects as well, but due to its flexibility, the cluster shape is continuously changing. Therefore, we categorize the trajectories by their (initial) average opening angle $\bar{\theta}$ of the smallest lag time interval and we use angular bins to summarize the results. The short-time diffusion tensor is calculated from experimental measurements in the following way:

$$
\boldsymbol{D}[i j](\bar{\theta}) \equiv \frac{1}{2} \phi_{i j} \frac{\partial\langle\Delta i \Delta j\rangle_{\tau}}{\partial \tau},
$$

with $\tau$ the lag time between frames, $\langle\cdots\rangle_{\tau}$ denotes a time average over all pairs of frames $\tau$ apart, and $\Delta i=i(t+$ $\tau)-i(t), \phi_{i j}$ is a correction factor that accounts for particleparticle and particle-substrate friction (see Methods section). The correction factors $\phi_{i j}$ are a first-order approximation to model the wall effect of the glass surface that, for translational diffusion, agrees closely with predictions from hydrodynamic theory (see Sec. B of the Supplemental Material Ref. [45]). We evaluated Eq. (3) at $\tau=0.25 s$, set by the frame rate of our camera.

Using Eq. (3), the resulting shape and time-dependent translational diffusivity in the $y$-direction of 12 rigid and one flexible trimer are shown in Fig. 1(d). Initially, at short timescales, there is a clear effect of cluster shape for both flexible and rigid trimers: translational diffusion in $y$ is highest for compact shapes. In comparison to rigid trimers, the diffusivity of the flexible trimer is slightly enhanced. Two other features unique to flexible clusters are that using a measurement of only one cluster, all possible cluster shapes are sampled, and the effect of shape vanishes on a much shorter timescale compared to the rigid clusters.

To study the diffusivity more carefully, we determined the average short time diffusion tensor of thirty flexible trimers. As shown in Fig. 2(a), the diffusion tensor elements were obtained by fitting the slope of the mean squared displacement versus lag time. We find three features that are in line with previous findings for rigid clusters [27] and that give confidence in the used analysis: first, translational diffusivity is higher along the longitudinal $x$ direction compared to the lateral $y$ direction [Fig. 2(d)]. Additionally, the rotational 

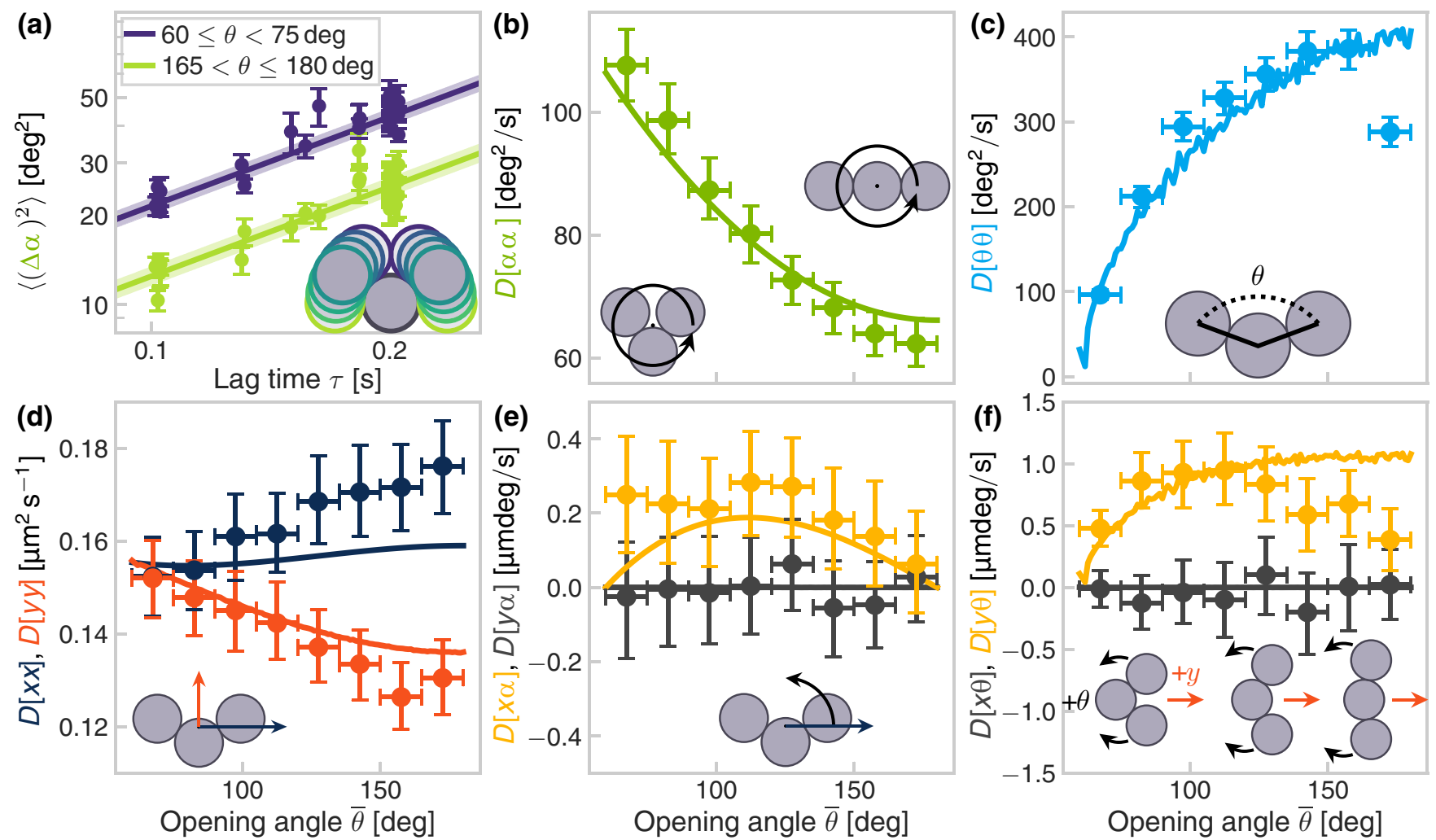

FIG. 2. Short-time translational, rotational, internal, and coupled diffusivity of flexible trimers (up to $0.25 \mathrm{~s}$ ). (a) Mean squared rotational displacements for lag times up to $\tau=0.25 \mathrm{~s}$, for two different instantaneous opening angles $\bar{\theta}$. (b) The rotational diffusivity is highest for the most compact shapes. (c) The joint flexibility increases as function of opening angle $\theta$. (d) While equal for flexed trimers, the translational diffusivity along the long axis $(x)$ is higher than along the short axis $(y)$. (e) We find a correlation between counterclockwise rotation and positive $x$ displacements. (f) There is a coupling between translational diffusion in the $y$-direction and shape changes: as the cluster diffuses in the positive $y$-direction, the angle $\theta$ increases, leading to a Brownian scallop-like motion at short timescales. In panels (b)-(f), the scatter points show the experimental measurements and the lines show the numerical calculations based on [31].

diffusivity shown in Fig. 2(b) is higher for compact trimers as opposed to fully extended trimers and we observe a coupling between translational diffusion and rotational diffusion in the $x$ direction [Fig. 2(e)].

However, flexibility gives rise to other modes that are not present in rigid assemblies. We found that the flexibility itself, as shown in Fig. 2(c), increases as a function of the opening angle, leading to a four-fold increase of flexibility for extended shapes compared to closed shapes. It is most likely caused by hydrodynamic interactions between the outer particles, as was predicted by earlier works [33].

Even more strikingly, the hydrodynamic drag on the outer particles leads to an increase in opening angle $\theta$ for positive displacements along the $y$ axis [Fig. 2(f)], which we call the Brownian quasiscallop mode. We stress that this correlation does not lead to self-propulsion because it has time-reversal symmetry. As the opening angle $\theta$ increases, the location of the center of mass moves in the negative $y$-direction of the original particle coordinate system. Therefore, this correlation is larger when the central particle is chosen as the origin of the coordinate center (see Sec. A of the Supplemental Material Ref. [45]). This Brownian quasiscallop mode may have implications for the accessibility of the functional site in induced fit lock-and-key interactions commonly observed in proteins [10].
Our experimental data allow us to test for the first time theoretical predictions made by Harvey and coworkers [31] who modelled the diffusion of segmentally flexible objects by calculating the hydrodynamic interactions between two sub units. We applied their calculations to a bead-shell model, adapted to match the conditions of our experiments (see the Methods and Sec. A of the Supplemental Material Ref. [45] for details), and found good agreement between the numerical calculations and the experimental data. The good agreement between the numerical results and the experimental data validates their model for the diffusivity of microscopic objects with internal degrees of freedom. For some angles and entries of the diffusion tensor, the experimental data show small deviations from the predicted model values, especially for translational diffusion, the Brownian quasiscallop mode, and the flexibility [see Figs. 2(c), 2(d) and 2(f)]. We hypothesize that these differences may arise because the numerical calculations do not take particle-particle and particle-substrate friction into account, other than as a first-order approximate scaling using the friction factors $\phi_{i j}$ as defined in Eq. (3). For example, substrate interactions were found to lead to enhanced diffusion for a model dumbbell consisting of two hydrodynamically coupled subunits [9]. More elaborate models may be used to provide higher-order corrections to the model we used here [64], however, their validity for flexible 
Flexible

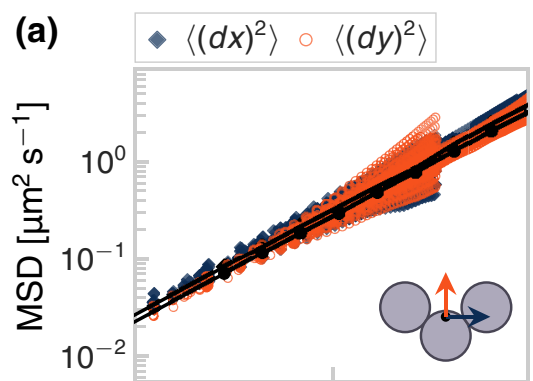

(c)

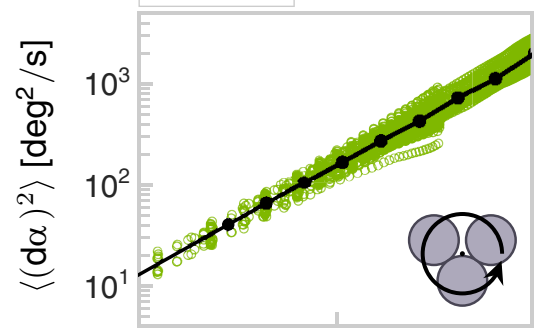

(e)

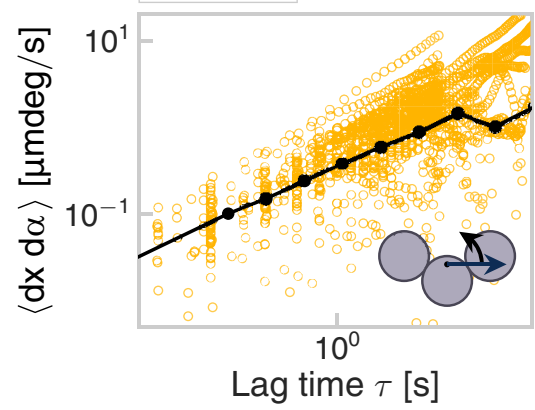

Flexible

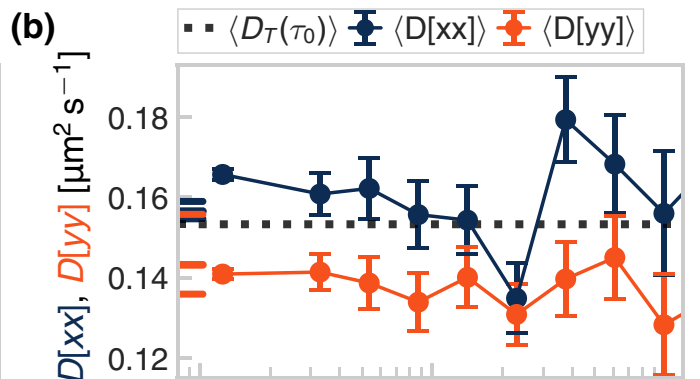

(d)
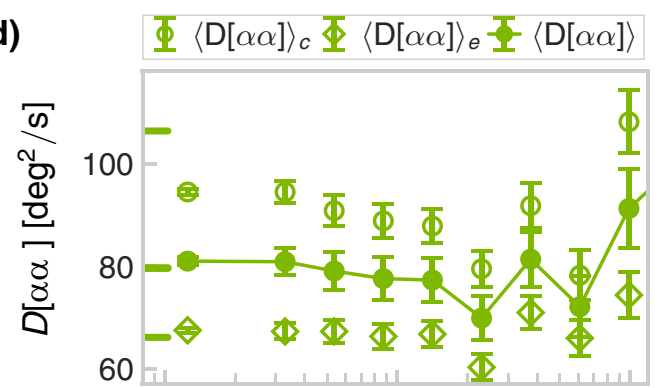

(f)

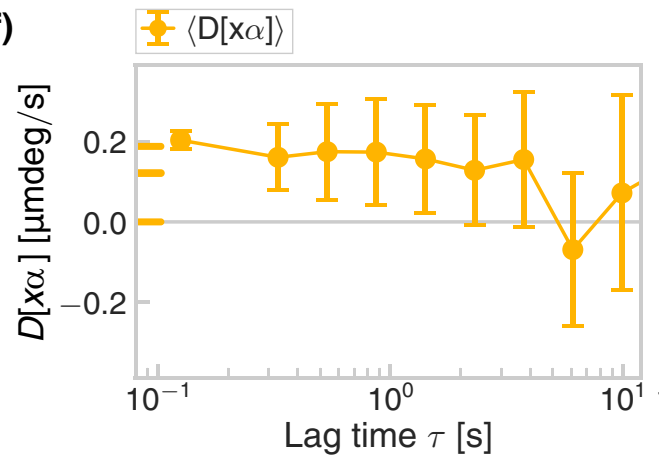

Rigid

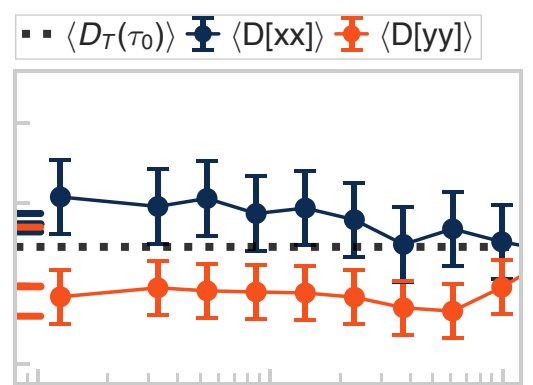

$\Phi\langle\mathrm{D}[\alpha \alpha]\rangle_{c} \Phi\langle\mathrm{D}[\alpha \alpha]\rangle_{e} \overline{\underline{T}}\langle\mathrm{D}[\alpha \alpha]\rangle$
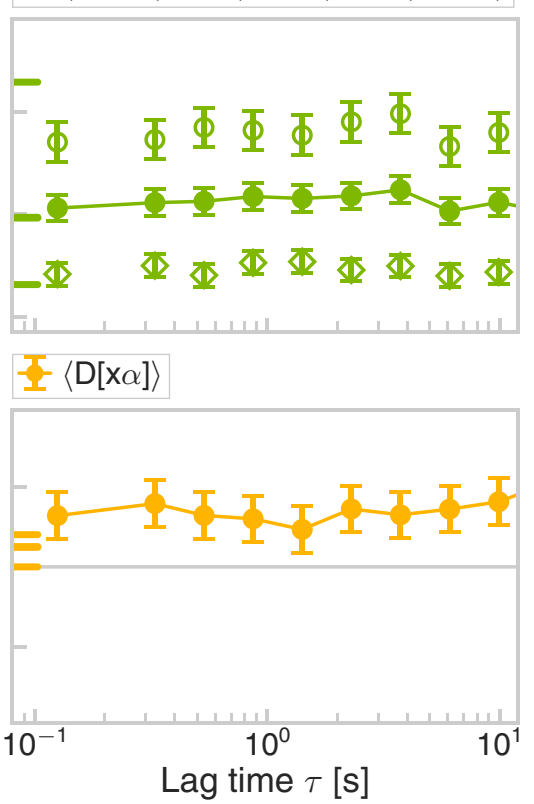

FIG. 3. Comparison between rigid and flexible trimers. (a) Mean squared displacements in $x$ and $y$ for all flexible trimers. (b) Diffusivity in $x$ and $y$ as function of lag time for flexible (left) and rigid (right) trimers. The average translational diffusivity $\left\langle D_{T}\left(\tau_{0}=0.25 \mathrm{~s}\right)\right\rangle(\mathrm{dotted}$ lines) is $(2.7 \pm 0.3) \%$ higher for flexible clusters compared to rigid clusters. (c) Mean squared angular displacements for all flexible trimers. (d) Rotational diffusivity as function of lag time for flexible (left) and rigid (right) trimers. $\langle\cdots\rangle_{c}$ correspond to $\bar{\theta}<120^{\circ}$ (compact) and $\langle\cdots\rangle_{e}$ to $\bar{\theta} \geqslant 120^{\circ}$ (extended). (e) Mean squared coupled displacements in $x$ and $\alpha$ for all flexible trimers. (f) Rotation-translation coupling in $x$ and $\alpha$ as function of lag time for flexible (left) and rigid (right) trimers. In panels (a), (c), and (e), colored points are experimental data, black points and lines represent the fitted slopes. In panels (b), (d), and (f), numerical short-time diffusivities calculated based on [31] are indicated by colored ticks on the $y$-axis, showing minimum, mean, and maximum shape-dependent values from bottom to top.

objects needs to be investigated. Moreover, our model also does not account for some out-of-plane diffusive motions against gravity that might occur in the experiments. Both effects are beyond the scope of our current work, but we hope they will be investigated in future studies.

Next, we compared the short-term translational, rotational, and coupled diffusion coefficients of flexible trimers to rigid trimers that are frozen in a particular shape and find that while they are qualitatively similar, there are experimentally measurable differences. Specifically, we measure that the average short time diffusion constant $\left\langle D_{T}\left(\tau_{0}=0.25 \mathrm{~s}\right)\right\rangle$ of rigid trimers is $(2.7 \pm 0.3) \%$ lower $[(15 \pm 2) \%$ lower without friction scaling] than that of flexible trimers [Figs. 3(a) and 3(b), dotted lines], a small but measurable effect corroborated by the numerical models (see Fig. S1 of the Supplemental Material Ref. [45]). The rotational diffusion constants for flexible and rigid trimers are equal within the experimental uncertainty [Figs. 3(c) and 3(d)], while the rotation-translation coupling mode between $x$ and $\alpha$ is slightly higher for flexible trimers at the shortest lag time [Figs. 3(e) and 3(f)]. These findings agree qualitatively with numerical predictions [11-13] for hinged chains of spheres of higher aspect ratio (20:1 instead of 3:1 for the trimers). For these hinged rods, a $10 \%$ increase in the translational diffusivity and a higher rotational diffusivity were found compared to rigid rods, which was attributed to hydrodynamic interactions between the subunits $[1,65]$.

The last way in which flexibility affects the diffusivity of a cluster is through the timescales on which effects of the initial cluster shape and orientation on the diffusive motions vanish. For rigid elongated particles it was shown that the timescale on which translational diffusivity in the $x$ and $y$ directions become equal with respect to the laboratory frame is set by the rotational diffusion time $\gamma_{r}=(D[\alpha \alpha])^{-1}$, with $D[\alpha \alpha]$ in $\mathrm{rad}^{2} / \mathrm{s}$ [21]. To study this effect for our rigid and freely jointed trimers, we analyzed the motion of the clusters by defining the laboratory frame in such a way that the center 
(a)

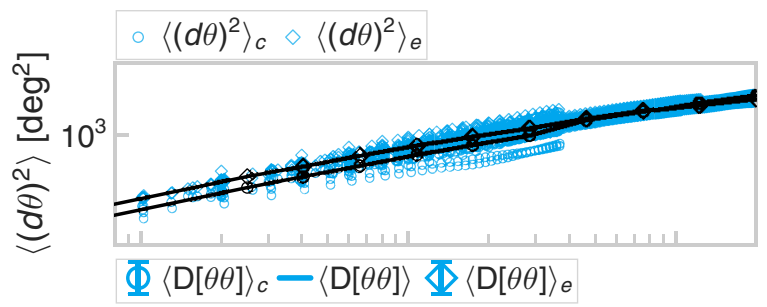

(b)

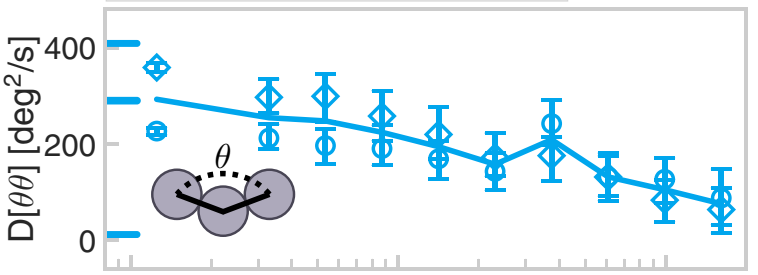

(c)

(d)
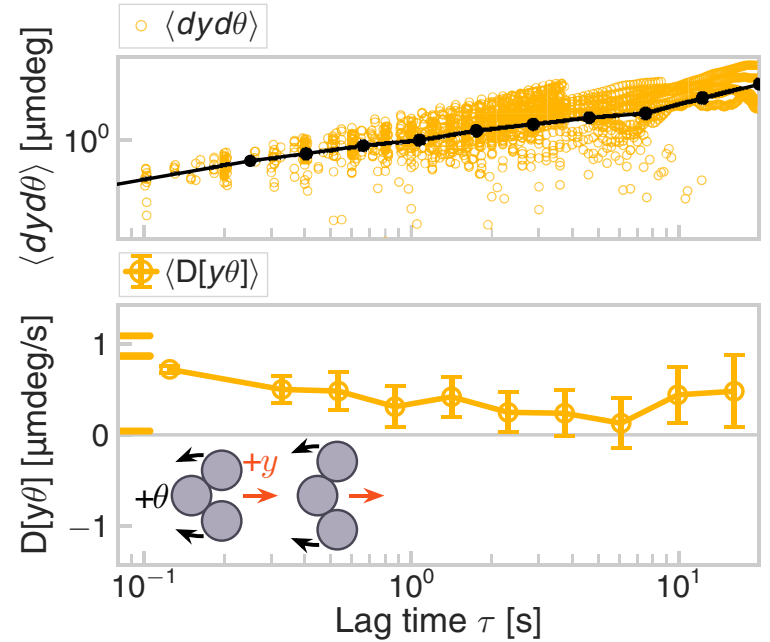

FIG. 4. Cluster flexibility and Brownian quasiscallop mode as function of time. (a) Mean squared angular displacements of $\theta$ for all flexible trimers. (b) The flexibility decreases as function of lag time because of hard-sphere repulsion between the two outer particles. (c) Mean squared coupled displacements of $y$ and $\theta$ for all flexible trimers. (d) The Brownian quasiscallop mode relaxes on a timescale of a few seconds because of conformational and rotational diffusion. In panels (a) and (b), $\langle\cdots\rangle_{c}$ correspond to $\bar{\theta}<120^{\circ}$ (compact) and $\langle\cdots\rangle_{e}$ to $\bar{\theta} \geqslant 120^{\circ}$ (extended). In panels (a) and (c), colored points are experimental data, black points and lines represent the fitted slopes. In panels (b) and (d), numerical short-time diffusivities calculated based on [31] are indicated by colored ticks on the $y$-axis, showing minimum, mean, and maximum shape-dependent values from bottom to top.

of mass of the trimer at lag time $\tau=0$ is at the origin and the body-centered $x$ and $y$ axes coincide with the original laboratory frame [see Fig. 1(c)], an approach inspired by earlier works on rigid anisotropic particles [24]. Using the values for the short time rotational diffusion coefficients for compact and extended trimers, we find that for both rigid and flexible trimers $30 \mathrm{~s} \leqslant \gamma_{r} \leqslant 60 \mathrm{~s}$. Indeed, by looking at the translational [Fig. 3(b)] diffusivity of rigid trimers, we see that the effect of shape on the diffusivity is preserved up to the maximum lag time we consider $(10 \mathrm{~s})$. The rotational diffusivity [Fig. 3(d)] of the rigid trimers stays constant within error (up to at least $10 \mathrm{~s}$ ).
However, for flexible trimers, the story is different. There exists a second timescale that can average out orientationdependent effects in diffusion: the timescale of shape changes, which we define as $\gamma_{s}=(D[\theta \theta])^{-1}$, analogous to the definition of the rotational diffusion time. Using the values for the short time flexibility coefficients for compact and extended trimers, we find that for our flexible trimers $8 \mathrm{~s} \leqslant$ $\gamma_{s} \leqslant 35 \mathrm{~s}$. Therefore, we hypothesize that for flexible trimers, internal deformations lead to faster relaxation of the shapedependency we observe at short lag times and therefore also the relaxation of differences between translational diffusion in the $x$ and $y$ directions.

Consistent with our hypothesis, the effect of the initial opening angle appears to be lost on a shorter timescale than what one would expect from the rotational diffusion time. In Fig. 3(d), the rotational diffusivity of flexible trimers is not constant in time, as is the case for rigid trimers, which shows that shape changes affect the diffusivity at longer lag times. The same effect can be seen in Fig. 4(b), where the cluster flexibility of compact and extended clusters become equal after about a second. Therefore, for lag times longer than $0.5 \mathrm{~s}$, we only consider the shape-averaged diffusivities. As can be seen from the translational diffusivity [Fig. 3(b)], the shape-averaged diffusivity in $x$ and $y$ become equal after 1 to $3 \mathrm{~s}$ and this is also the timescale on which the rotational diffusivity is no longer constant [Fig. 3(d)] and the translation-rotation coupling vanishes [Fig. 3(f)]. Moreover, we observe for both translational, rotational, and translationrotation coupled diffusion that after lag times larger than $2 \mathrm{~s}$, larger fluctuations occur which we attribute to the effect of continuous shape-changes [see Figs. 3(b), 3(d) and 3(f)].

Short timescale relaxation of differences between clusters in extended and compact conformations exist also for the conformational diffusion tensor elements. The flexibility [shown in Figs. 4(a) and 4(b)] is smaller for trimers in flexed conformations than in extended conformations and the difference vanishes after approximately $2 \mathrm{~s}$ due to shape changes. Figure 4(b) shows an overall decrease of flexibility with lag time because the range wherein the joint angle can vary is bounded by the two outermost particles. Furthermore, the magnitude of $D[y \theta]$ [shown in Figs. 4(c) and 4(d)], which represents the Brownian quasiscallop mode, vanishes on the same timescale of approximately $2 \mathrm{~s}$, set by the conformational relaxation time $8 \mathrm{~s} \leqslant \gamma_{s} \leqslant 35 \mathrm{~s}$.

\section{CONCLUSION}

In conclusion, we studied the Brownian motion of flexible trimers and found features that are unique to flexible objects. We found a hydrodynamic coupling between conformational changes and translations perpendicular to the particle's long axis ( $y$-direction), which we call the Brownian quasiscallop mode because of its resemblance to scallop propulsion at high Reynolds numbers. We found that this coupling persists over several seconds, a timescale relevant for biomolecular interactions, implying that it might affect the association of flexible proteins and other biomolecules. Second, we found that the long-time translational diffusion of the freely jointed trimers was 3 to $15 \%$ higher than that of their rigid counterparts. This enhancement was predicted for hinged rods [11-13], but contrasts with theoretical results on dumbbells of two hydrodynamically coupled subunits, in which extensile shape 
fluctuations were shown to decrease the translational diffusion coefficient $[9,14]$. Further theoretical and experimental studies are needed to predict the effect of flexibility on diffusivity since different internal degrees of freedom can have opposing effects. Finally, we showed that the transition from shortto long-time diffusion depends not (only) on the rotational diffusion time but mainly on a timescale related to conformational changes of the particle. We were able to describe our experimental findings using a hydrodynamic modeling procedure that combines bead-shell modeling with the approach of Harvey and coworkers [31]. We hope this work inspires other researchers to more confidently apply this method in the context of the diffusion of segmentally flexible systems such as biopolymers and proteins.

\section{ACKNOWLEDGMENTS}

This project received funding from the European Research Council (ERC) under the European Union's Horizon 2020 research and innovation program (Grant No. 758383) and from the NWO graduate program.

Author Contributions. R.W.V. and P.G.M. contributed equally to the work. P.G.M., R.W.V., N.E.G.L., and L.P.P.H. performed the experiments. P.G.M. and R.W.V. analysed the data. R.W.V. performed the hydrodynamic modeling. P.G.M., R.W.V., J.G., W.K.K., A.v.B., and D.J.K. conceived of the experiments. P.G.M., R.W.V., W.K.K., A.v.B., and D.J.K. wrote the paper. All authors discussed the results and contributed to the final article.
[1] P. Mellado, A. Iniesta, F. G. Diaz, and J. Garcia de la Torre, Diffusion coefficients of segmentally flexible macromolecules with two subunits: A study of broken rods, Biopolymers 27, 1771 (1988).

[2] S. Ishino, T. Yamagami, M. Kitamura, N. Kodera, T. Mori, S. Sugiyama, T. Ando, N. Goda, T. Tenno, H. Hiroaki, and Y. Ishino, Multiple interactions of the intrinsically disordered region between the helicase and nuclease domains of the archaeal Hef protein, J. Biol. Chem. 289, 21627 (2014).

[3] J. Yguerabide, H. F. Epstein, and L. Stryer, Segmental flexibility in an antibody molecule, J. Mol Biol. 51, 573 (1970).

[4] J. Garcia de la Torre, Hydrodynamics of segmentally flexible macromolecules, Eur. Biophys. J. 23, 307 (1994).

[5] D. R. Burton, Structure and function of antibodies, in New Comprehensive Biochemistry, Vol. 17 (Elsevier, Amsterdam, 1987), pp. 1-50.

[6] G. Barbato, M. Ikura, L. E. Kay, R. W. Pastor, and A. Bax, Backbone dynamics of calmodulin studied by nitrogen- 15 relaxation using inverse detected two-dimensional NMR spectroscopy: the central helix is flexible, Biochemistry 31, 5269 (1992).

[7] I. N. Serdyuk, N. R. Zaccai, and J. Zaccai, Methods in Molecular Biophysics: Structure, Dynamics, Function (Cambridge University Press, Cambridge, England, 2007).

[8] L. Gregory, K. G. Davis, B. Sheth, J. Boyd, R. Jefferis, C. Nave, and D. R. Burton, The solution conformations of the subclasses of human IgG deduced from sedimentation and small angle $\mathrm{X}$ ray scattering studies, Mol. Immunol. 24, 821 (1987).

[9] P. Illien, T. Adeleke-Larodo, and R. Golestanian, Diffusion of an enzyme: The role of fluctuation-induced hydrodynamic coupling, Europhys. Lett. 119, 40002 (2017).

[10] D. E. Koshland, The Key-Lock Theory and the Induced Fit Theory, Angew. Chem. Int. Ed. Engl. 33, 2375 (1995).

[11] K. Nagasaka and H. Yamakawa, Dynamics of weakly bending rods: A trumbbell model, J. Chem. Phys. 83, 6480 (1985).

[12] M. Fixman, Inclusion of hydrodynamic interaction in polymer dynamical simulations, Macromolecules 14, 1710 (1981).

[13] A. Z. Akcasu, Comments on the diffusion coefficient and first cumulant, Macromolecules 15, 1321 (1982).

[14] T. Adeleke-Larodo, P. Illien, and R. Golestanian, Fluctuationinduced hydrodynamic coupling in an anisotropic dumbbell, Eur. Phys. J. E 42, 11799 (2019).

[15] C. Riedel, R. Gabizon, C. A. M. Wilson, K. Hamadani, K. Tsekouras, S. Marqusee, S. Press, and C. Bustamante, The heat released during catalytic turnover enhances the diffusion of an enzyme, Nature 517, 227 (2015).

[16] S. Sengupta, K. K. Dey, H. S. Muddana, T. Tabouillot, M. E. Ibele, P. J. Butler, A. Sen, Enzyme molecules as nanomotors, J. Am. Chem. Soc. 135, 1406 (2013).

[17] L. Rundqvist, J. Adén, T. Sparrman, M. Wallgren, U. Olsson, and M. Wolf-Watz, Noncooperative Folding of Subdomains in Adenylate Kinase, Biochemistry 48, 1911 (2009).

[18] A. Einstein, Über die von der molekularkinetischen Theorie der Wärme geforderte Bewegung von in ruhenden Flüssigkeiten suspendierten Teilchen, Ann. Phys. (Leipzig) 322, 549 (1905).

[19] W. Sutherland, A dynamical theory of diffusion for nonelectrolytes and the molecular mass of albumin, Philos. Mag. Series 6, 9, 781 (1905).

[20] J. Perrin, Mouvement brownien et réalité moléculaire, Ann. Chim. Phys. 18, 5 (1909).

[21] Y. Han, A. M. Alsayed, M. Nobili, J. Zhang, T. C. Lubensky, and A. G. Yodh, Brownian motion of an ellipsoid, Science 314, 626 (2006).

[22] A. Meunier, Friction coefficient of rod-like chains of spheres at very low Reynolds numbers. II. Numerical simulations, J. de Phys. Archives 4, 561 (1994).

[23] K. Zahn, R. Lenke, and G. Maret, Friction coefficient of rod-like chains of spheres at very low Reynolds numbers. I. Experiment, J. Phys. II France 4, 555 (1994).

[24] A. Chakrabarty, A. Konya, F. Wang, J. V. Selinger, K. Sun, and Q. Wei, Brownian motion of arbitrarily shaped particles in two dimensions, Langmuir 30, 13844 (2014).

[25] A. Chakrabarty, F. Wang, K. Sun, and Q. Wei, Effects of translation - rotation coupling on the displacement probability distribution functions of boomerang colloidal particles, Soft Matter 12, 4318 (2016).

[26] L. Koens, M. Lisicki, and E. Lauga, The non-Gaussian tops and tails of diffusing boomerangs, Soft Matter 13, 2977 (2017).

[27] D. J. Kraft, R. Wittkowski, B. Ten Hagen, K. V. Edmond, D. J. Pine, and H. Löwen, Brownian motion and the hydrodynamic friction tensor for colloidal particles of complex shape, Phys. Rev. E 88, 050301 (2013).

[28] J. Fung, and V. Manoharan, Holographic measurements of anisotropic three-dimensional diffusion of colloidal clusters, Phys. Rev. E 88, 20302 (2013).

[29] M. Hoffmann, C. S. Wagner, L. Harnau, and A. Wittemann, 3D Brownian diffusion of submicron- sized particle clusters, ACS Nano 3, 3326 (2009). 
[30] A. V. Butenko, E. Mogilko, L. Amitai, B. Pokroy, and E. Sloutskin, Coiled to diffuse: Brownian motion of a helical bacterium, Langmuir 28, 12941 (2012).

[31] S. C. Harvey, P. Mellado, and J. Garcia de la Torre, Hydrodynamic resistance and diffusion coefficients of segmentally flexible macromolecules with two subunits, J. Chem. Phys. 78, 2081 (1983).

[32] D. B. Roitman, The Elastic Trumbbell Model for Dynamics of Stiff Chains, in Rotational Dynamics of Small and Macromolecules, Lecture Notes in Physics, Vol. 293 (Springer, Berlin, 2005).

[33] W. A. Wegener, Bead models of segmentally flexible macromolecules, J. Chem. Phys. 76, 6425 (1982).

[34] W. A. Wegener, Center of diffusion of flexible macromolecules, Macromolecules 18, 2522 (1985).

[35] J. Garcia de la Torre and B. Carrasco, Hydrodynamic properties of rigid macromolecules composed of ellipsoidal and cylindrical subunits, Biopolymers 63, 163 (2002)..

[36] A. Iniesta, F. G. Diaz, and J. Garcia de la Torre, Transport properties of rigid bent-rod macromolecules and of semiflexible broken rods in the rigid-body treatment, Analysis of the flexibility of myosin rod, Biophys. J. 54, 269 (1988).

[37] S. A. J. Van der Meulen and M. E. Leunissen, Solid colloids with surface-mobile DNA linkers, J. Am. Chem. Soc. 135, 15129 (2013).

[38] S. A. J. Van der Meulen, G. Helms, and M. Dogterom, Solid colloids with surface-mobile linkers, J. Phys.: Condens. Matter 27, 233101 (2015).

[39] I. Chakraborty, V. Meester, C. van der Wel, and D. J. Kraft, Colloidal joints with designed motion range and tunable joint flexibility, Nanoscale 9, 7814 (2017).

[40] Y. Zhang, A. Mcmullen, L. Pontani, X. He, R. Sha, N. C. Seeman, J. Brujic, and P. M. Chaikin, Sequential self-assembly of DNA functionalized droplets, Nat. Commun. 8, 1 (2017).

[41] Y. Zhang, X. He, R. Zhuo, R. Sha, J. Brujic, N. C. Seeman, and P. M. Chaikin, Multivalent, multiflavored droplets by design, Proc. Natl. Acad. Sci. USA 115, 9086 (2018).

[42] A. Mcmullen, M. Holmes-Cerfon, F. Sciortino, A. Y. Grosberg, and J. Brujic, Freely Jointed Polymers Made of Droplets, Phys. Rev. Lett. 121, 138002 (2018).

[43] M. Rinaldin, R. W. Verweij, I. Chakraborty, and D. J. Kraft, Colloid supported lipid bilayers for self-assembly, Soft Matter 15, 1345 (2019).

[44] H. R. Vutukuri, A. F. Demirörs, B. Peng, P. D. J. van Oostrum, A. Imhof, and A. van Blaaderen, Colloidal analogues of charged and uncharged polymer chains with tunable stiffness, Angew. Chem., Int. Ed. 51, 11249 (2012).

[45] See Supplemental Material at http://link.aps.org/supplemental/ 10.1103/PhysRevResearch.2.033136 for consists of a movie of a flexible trimer as well as details on the hydrodynamic models (Sec. A) and friction factors (Sec. B), the effect of size polydispersity on the collective diffusion coefficient (Sec. C), the opening angle probability distribution for flexible trimers (Fig. S5), angles of the rigid trimers and their diffusion (Figs. S6 and S7), plus an overview of all the DNA strands used here (Table S1).

[46] C. Van der Wel, A. Vahid, A. Saric, T. Idema, D. Heinrich, and D. J. Kraft, Lipid membrane-mediated attrac- tion between curvature inducing objects, Sci. Rep. 6, 32825 (2016).

[47] D. Allan, C. Van der Wel, N. Keim, T. A. Caswell, D. Wieker, R. W. Verweij, C. Reid et al., TRACKPY: a general-purpose PYTHON tracking library (v0.4.2), Zenodo (2019).

[48] M. E. Leunissen, R. Dreyfus, R. Sha, T. Wang, N. C. Seeman, D. J. Pine, and P. Chaikin, Towards self-replicating materials of DNA-functionalized colloids, Soft Matter 5, 2422 (2009).

[49] F. Montanarella, D. Urbonas, L. Chadwick, P. Moerman, P. Beasjou, J. Patrick, R. Mahrt, A. van Blaaderen, T. Stöferle, and D. Vanmaekelbergh, Lasing supraparticles self-assembled from nanoparticles, ACS Nano 12, 12788 (2018).

[50] J. Crocker and D. Grier, Methods of digital microscopy for colloidal studies, J. Colloid Interface Sci. 179, 298 (1996).

[51] J. T. Padding and W. J. Briels, Translational and rotational friction on a colloidal rod near a wall, J. Chem. Phys. 132, 054511 (2010).

[52] M. Newville, T. Stensitzki, D. B. Allen, and A. Ingargiola, LMFIT: Non-Linear least-square minimization and curve-fitting for python, Zenodo (2014).

[53] D. Foreman-Mackey, D. W. Hogg, D. Lang, and J. Goodman, emcee: The MCMC Hammer, Publ. Astron. Soc. Pac. 125, 306 (2013).

[54] F. G. Diaz and J. Garcia de la Torre, Viscoelastic properties of semiflexible macromolecules in solution: Brownian dynamics simulation of a trumbbell model, Macromolecules 27, 5371 (1994).

[55] B. Carrasco and J. Garcia de la Torre, Hydrodynamic properties of rigid particles: comparison of different modeling and computational procedures, Biophys J. 76, 3044 (1999).

[56] V. Bloomfield, W. O. Dalton, and K. E. Van Holde, Frictional coefficients of multisubunit structures. I. Theory, Biopolymers 5, 135 (1967).

[57] D. P. Filson and V. Bloomfield, Shell model calculations of rotational diffusion coefficients, Biochemistry 6, 1650 (1967).

[58] D. L. Ermak and J. A. McCammon, Brownian dynamics with hydrodynamic interactions, J. Chem. Phys. 69, 1352 (1978).

[59] J. Rotne and S. Prager, Variational treatment of hydrodynamic interaction in polymers, J. Chem. Phys. 50, 4831 (1969).

[60] H. Yamakawa, Transport properties of polymer chains in dilute solution: Hydrodynamic interaction, J. Chem. Phys. 53, 436 (1970).

[61] B. Carrasco and J. Garcia de la Torre, Improved hydrodynamic interaction in macromolecular bead models, J. Chem. Phys. 111, 4817 (1999).

[62] G. D. J. Phillies, Translational drag coefficients of assemblies of spheres with higher-order hydrodynamic interactions, J. Chem. Phys. 81, 4046 (1984).

[63] J. Happel and H. Brenner, Low Reynolds Number Hydrodynamics: With Special Applications to Particulate Media (Springer Science \& Business Media, New York, 2012).

[64] J. W. Swan, and J. F. Brady, Simulation of hydrodynamically interacting particles near a no-slip boundary, Phys. Fluids 19, 113306 (2007).

[65] S. C. Harvey, Transport properties of particles with segmental flexibility. I. Hydrodynamic resistance and diffusion coefficients of a freely hinged particle, Biopolymers 18, 1081 (1979). 